\title{
AMPLIAÇÃO DA DISTRIBUIÇÃO DAS ANÊMONAS-DO-MAR (CNIDARIA, ACTINIARIA) NO ESTADO DE PERNAMBUCO, BRASIL.
}

\author{
Katarina VASCONCELOS DE MELO ${ }^{1}$ \\ Fernanda DUARTE AMARAL ${ }^{2}$
}

Departamento de Biologia, Área de Zoologia, Laboratório de Ambientes Recifais, Universidade Federal Rural de Pernambuco; ${ }^{1}$ katyvmelo@ig.com.br ${ }^{2}$ fdamaral@ufrpe.br

\author{
Recebido: $14 / 07 / 2002$
}

Aceito: 20/03/2005

\section{RESUMO}

O objetivo deste estudo foi realizar um levantamento qualitativo e descrever as espécies de anêmonas das praias de Boa Viagem, Muro Alto, Carneiros e Forno da Cal, no Estado de Pernambuco. As anêmonas foram coletadas, no período de 12 de agosto de 2000 a 11 de novembro de 2001, com o auxílio da espátula, ponteira e martelo e foram armazenadas em sacos plásticos com água do mar, devidamente etiquetados, e levadas ao Laboratório de Ambientes Recifais (LAR/UFRPE), onde foram anestesiadas com $20 \mathrm{~g}$ de cloreto de magnésio diluídos em $500 \mathrm{ml}$ de água salgada, sendo, posteriormente, fixadas em formol a 10\%. Foram analisadas primeiramente a coloração e as estruturas morfológicas do corpo das anêmonas, assim como mensuradas, com o auxílio do paquímetro e da ocular micrométrica, a altura e o diâmetro da coluna, do disco oral e basal e contado o número total de tentáculos para cada exemplar. Posteriormente, com o estereomicroscópio foram analisados os caracteres anatômicos como: ciclos de mesentérios, pares de diretivos, sifonoglifes, filamentos mesentéricos e gônadas, assim como também algumas cnidas foram analisadas e identificadas. Foram coletadas e inventariadas até o momento seis espécies: Bunodosoma cangicum Corrêa,1964 in Belém \& Preslercravo, 1973, Anthopleura cascaia Corrêa,1964 in Dube, 1981, Anthopleura krebsi Duch. \& Mich., 1860, Anthopleura sp., Anemonia sp., e Phyllactis flosculifera (Lesueur, 1817), sendo quatro destas relatadas para a Praia de Boa Viagem, três para a Praia de Muro Alto, três para a Praia dos Carneiros e três para a Praia de Forno da Cal. Ainda foram observados na Praia dos Carneiros, exemplares do gênero Bellactis e algumas outras Acontiaria, perfazendo um total de sete espécies de Actiniaria identificadas. A morfologia, a anatomia e os cnidocistos de cada espécie foram comparados com os resultados da literatura. Observou-se uma grande variação de alguns caracteres morfológicos, tais como a coloração.

Palavras chaves: anêmonas-do-mar; morfologia; anatomia; biodiversidade; Boa Viagem; Muro Alto; Carneiros; Forno da Cal. 


\section{ABSTRACT \\ Distribution Enlargement of Some Sea Anemones (Cnidaria, Actiniaria) in the State of Pernambuco, Brazil.}

The aim of this study was to perform a qualitative survey and a description of the sea anemones of the beaches of Boa Viagem, Muro Alto, Carneiros, and Forno da Cal, all in the state of Pernambuco (Brazil). The sea anemones were collected between August 12, 2000 and November 11, 2001 with the aid of a scoop, crampon, and hammer. The specimens were stored in labeled plastic bags filled with seawater and taken to the Reef Environment Laboratory (Laboratório de Ambientes Recifais - LAR/UFRPE), where they were anesthetized with $20 \mathrm{~g}$ of magnesium chloride diluted in $500 \mathrm{ml}$ of seawater and later fixed with formaldehyde at $10 \%$. The coloring and morphological structures of the sea anemones were the first characters to be analyzed; some structures were also measured (with the aid of a caliper and a microscope with an ocular micrometer): the height and diameter of the column and of the oral and basal discs, as well as the number of tentacles of some specimens of each species. In addition, a stereomicroscope was used to analyze anatomical characters such as: mesentery cycles, directives pairs, siphonoglyphs, mesenteric filaments, and gonads. Some cnidae were also analyzed and identified. Up to now, six species were collected and studied: Bunodosoma cangicum Corrêa,1964 in Belém \& Preslercravo, 1973 Anthopleura cascaia Corrêa,1964 in Dube, 1981, Anthopleura krebsi Duch. \& Mich., 1860, Anthopleura sp., Anemonia sp., and Phyllactis flosculifera (Lesueur, 1817), from which four were found in Boa Viagem Beach, three in Muro Alto Beach, three in Carneiros Beach, and three in Forno da Cal Beach. In addition, specimens of the genus Bellactis and some other Acontiaria were observed in Carneiros Beach - a total of seven Actiniaria species were identified during this study. The morphology, anatomy, and cnidae of each species were compared to the results found in the literature. Great variation of some morphological characters was observed, such as coloring.

Key words: Sea anemones, morphology, anatomy, biodiversity, Boa Viagem, Muro Alto, Carneiros, Forno da Cal.

\section{INTRODUÇÃO}

As anêmonas-do-mar são pólipos solitários que fazem parte do Filo Cnidaria, Classe Anthozoa, Ordem Actiniaria ou Corallimorpharia e são consideradas de fundamental importância para a ecologia, pois fazem parte da cadeia alimentar, apresentam relações simbióticas com vários outros animais e possuem freqüentemente em seus tecidos zooxantelas que participam na produtividade nos recifes (FAUTIN \& ALLEN, 1992). Por serem animais bentônicos estão sujeitas a fatores externos como: tipo de substrato, modo de ação das ondas, mudança de marés, entre outros. Estes fatores vão condicionar a sua distribuição (GOMES et al., 1998).

As anêmonas são freqüentemente encontradas em ambientes recifais: substratos duros colonizados por uma fauna coralínea razoável (com uma considerável biodiversidade de animais e plantas e uma enorme importância ecológica e econômica), constituindo um ecossistema recifal (AMARAL et al., 2003; AMARAL \& STEINER, 2002).

As anêmonas possuem grande resistência a variações no meio em que vivem, tornando-se importantes indicadores de poluição. Além disso, algumas espécies (como Bunodosoma caissarum 
Corrêa in Belém, 1988) são estudadas quanto à existência de substâncias bioativas (FREITAS \& SAWAYA, 1990 apud MIGOTTO et al., 1999).

Os trabalhos aprofundados sobre anemonofauna de Pernambuco tiveram seu início na década de 90, com os trabalhos de Gomes (1996) e Gomes \& Mayal (1997), onde estas autoras registraram oito espécies. Posteriormente, Gomes \& Zamponi (1998) registraram a ocorrência de Phyllactis correae Schlenz \& Belém, 1988 para o litoral pernambucano. Segundo Gomes (2002), para o setor continental pernambucano são conhecidas 13 espécies, porém se considerarmos o Arquipélago de Fernando de Noronha este número se eleva para 17.

A iniciativa de se fazer um levantamento deste grupo nas praias de Boa Viagem, Muro Alto, Carneiros e Forno da Cal deve-se ao fato destes locais apresentarem uma riqueza em quantidade de anêmonas, como também porque propiciará um maior conhecimento da anemonofauna e a identificação de possíveis novas espécies.

O objetivo deste estudo foi realizar um levantamento qualitativo, descrever a morfologia dos caracteres e analisar o cnidoma das espécies de anêmonas das praias de Boa Viagem, Muro Alto, Carneiros e Forno da Cal, em Pernambuco, Brasil.

\section{MATERIAL E MÉTODOS}

As anêmonas foram coletadas aleatoriamente em maré baixa, no mediolitoral, entre as locas e frestas do substrato rochoso ou em bancos de arenitos das praias de Boa Viagem, Muro Alto, Carneiros e Forno da Cal, em profundidades que variaram de zero a dois metros, no período de 12 de agosto de 2000 a 11 de novembro de 2001, com auxílio da espátula, ponteira e martelo. Os espécimes foram armazenados em sacos plásticos com água do mar devidamente etiquetados contendo local, data e dados de coleta, sendo posteriormente levados ao Laboratório de Ambientes Recifais da Universidade Federal Rural de Pernambuco (LAR/UFRPE).

No LAR/UFRPE, foram observadas in vivo em um aquário, onde as seguintes características externas foram anotadas: a coloração, as estruturas (verrugas, vesículas, esférulas marginais, disco pedal, tentáculos, disco oral, presença ou não de acôncios, etc.) e a forma do corpo do animal.

Posteriormente, o material foi anestesiado através de uma solução com $20 \mathrm{~g}$ de $\mathrm{MgCl}_{2}$ dissolvido em $500 \mathrm{ml}$ de água salgada (adicionando-se vagarosamente no recipiente). Os espécimes foram fixados em formol a $10 \%$ e tombados na coleção do LAR/UFRPE.

O estudo macroscópico foi realizado com o auxílio de um paquímetro e de um estereomicróscopio com ocular micrométrica. Foram mensurados: altura e diâmetro da coluna, do disco oral e do disco pedal e contados o número total de tentáculos. Através de cortes transversais, com lâmina na altura da coluna dos espécimes, foram feitas anotações quanto ao número de ciclos mesentéricos, pares de diretivos e sifonoglifes, músculos retratores, filamentos mesentéricos e gônadas, com o auxílio do estereomicroscópio. Foram calculados a média e o desvio padrão dos caracteres morfológicos estudados nas espécies de anêmonas coletadas e identificadas para as quatro localidades do Estado de Pernambuco.

Cortes de várias estruturas foram realizados nas espécies estudadas, tais como: tentáculos, esférulas, coluna, actinofaringe e filamentos mesentéricos, e de cada pedaço retirado foi feito um esfregaço em lâmina e colocada uma gota de água para se visualizar e identificar os cnidocistos com auxílio de um microscópio óptico na objetiva de 100x. Para a identificação dos cnidocistos foi utilizado Schmidt (1974).

Tropical Oceanography, Recife, v. 33, n. 1, p. 19-31, 2005. 


\section{CARACTERIZAÇÃO DOS LOCAIS DE ESTUDO}

As praias estudadas no presente trabalho foram as seguintes:

Praia de Boa Viagem (Recife) - A Praia de Boa Viagem, principal praia da cidade do Recife, situa-se entre os paralelos $08^{\circ} 06^{\prime} 02^{\prime \prime}$ e $08^{\circ} 08^{\prime} 06^{\prime \prime}$ de latitude Sul e meridianos $34^{\circ} 52^{\prime} 48^{\prime \prime}$ e $34^{\circ} 53^{\prime} 47^{\prime \prime}$ de longitude Oeste. Os recifes de Boa Viagem são principalmente bancos de arenito e se apresentam em linhas paralelas à costa, alguns dos quais são totalmente ou parcialmente emersos, durante a maré baixa (LUZ, 1991 apud STEINER, 2003).

Muro Alto (Ipojuca) - está situada no litoral Sul de Pernambuco, no município de Ipojuca, a $65 \mathrm{Km}$ da cidade do Recife. Possui as seguintes coordenadas geográficas: $08^{\circ} 06^{\prime}$ e $08^{\circ} 08^{\prime} \mathrm{S}$ e $34^{\circ}$ $52^{\prime}$ e $34^{\circ} 53^{\prime}$ W. Esta praia possui um mediolitoral onde predominam bancos de arenito e sofre uma grande pressão antrópica por ser um pólo turístico.

Praia dos Carneiros (Tamandaré) - Esta praia pertence ao município de Tamandaré e está situada a cerca de $110 \mathrm{~km}$ ao Sul da cidade do Recife, seus limites são o rio Formoso (ao Norte) e o rio Mamucaba (ao sul) e possui as seguintes coordenadas geográficas $08^{\circ} 47^{\prime} 20^{\prime}$ ' $S$ e $35^{\circ} 06^{\prime} 45^{\text {'’ }} \mathrm{W}$, totalizando uma área de $9 \mathrm{Km}$ de extensão. Os recifes de Tamandaré estão assentados sobre bancos de arenito a apenas $1 \mathrm{Km}$ da praia. São recifes alongados, distribuídos em três linhas paralelas à costa e chamados classicamente de recifes em franja (AMARAL, 1986).

Praia de Forno da Cal (Itamaracá) - Possui as seguintes coordenadas geográficas: latitude 747’S e longitude 3450'W (LIRA, 1975 apud ALVES, 2000). Foram estudadas a Pedra do Jacaré e a Pedra do Anel (formações rochosas do mediolitoral com o formato de jacaré e de anel, respectivamente).

\section{RESULTADOS}

Foram identificadas sete espécies de anêmonas: Bunodosoma cangicum Corrêa, 1964 in Belém \& Preslercravo, 1973, Anthopleura cascaia Corrêa, 1964 in Dube, 1981, Anthopleura krebsi Duch. \& Mich., 1860, Anthopleura sp., Anemonia sp. Phyllactis flosculifera (Lesueur, 1817) e Bellactis sp., sendo esta última apenas observada. (Tabela 1).

Tabela 1- Distribuição das espécies de anêmonas estudadas para o litoral de Pernambuco, coletadas e/ou observadas no período de 12 de agosto de 2000 a 11 de novembro de 2001.

\begin{tabular}{lllll}
\hline Espécies & Boa Viagem & Muro Alto & Carneiros & Forno da Cal \\
\hline Bunodosoma cangicum & $\mathrm{X}$ & $\mathrm{X}$ & $\mathrm{X}$ & $\mathrm{X}$ \\
Anthopleura cascaia & $\mathrm{X}$ & & & $\mathrm{X}$ \\
Anthopleura krebsi & $\mathrm{X}$ & $\mathrm{X}$ & $\mathrm{X}$ & \\
Anthopleura sp. & $\mathrm{X}$ & & & $\mathrm{X}$ \\
Anemonia sp. & & & $\mathrm{X}$ & \\
Phyllactis flosculifera & & $\mathrm{X}$ & $\mathrm{X}$ & \\
Bellactis sp. & & & & \\
\hline
\end{tabular}

Bunodosoma cangicum Corrêa, 1964 in Belém \& Preslercravo, 1973 (Figura 1A).

Tropical Oceanography, Recife, v. 33, n. 1, p. 19-31, 2005. 
Distribuição geográfica:

Brasil - ES e RJ: Belém \& Preslercravo (1973); SP: Corrêa (1964); BA: Dube (1974); no Arquipélago de São Pedro e São Paulo (AMARAL et al., 2002) e PE.

Pernambuco - Bairro Novo, Boa Viagem, Piedade, Enseada dos Corais, Serrambi, Carneiros, Muro Alto e Forno da Cal: Gomes (1996), Gomes \& Mayal (1997) e o presente estudo.

Morfologia externa:

Anêmona comparativamente grande em relação as demais espécies identificadas no presente estudo. Disco pedal: arredondado, bem expandido (diâmetro de 2,45 \pm 0,62 cm) e aderente com estrias que são as inserções mesenteriais; apresenta cor alaranjada, parda, marrom, cinza ou avermelhada. Coluna: em forma de cilindro (diâmetro de 2,6 $\pm 1,2 \mathrm{~cm}$ ), com coloração laranja ou avermelhada e com vesículas dispostas em torno de toda a coluna nas cores verdes, alaranjadas, avermelhadas, creme ou amarronzadas, esférulas marginais numa série de 48, esverdeadas a esbranquiçadas ou alaranjadas e inserções mesenteriais bem visíveis. Tentáculos: número total de 96, compridos, simples e numerosos, pontiagudos, com reflexos de coloração laranja, esverdeada, avermelhada ou parda. Disco oral: (diâmetro de 2,42 \pm 0,79 cm) com boca grande, bem visível e escura, amarronzada, acinzentada e esverdeada com reflexos verdes e laranja (Tabela 2).

Tabela 2 - Média e ( \pm ) desvio padrão dos caracteres morfológicos estudados em seis espécies de anêmonas de Pernambuco. Material coletado no período de 12 de agosto de 2000 a 11 de novembro de 2001. N. exemp. = número total de exemplares coletados; $\mathrm{n}$ = número de exemplares analisados para cada estrutura. Medidas em centímetros.

\begin{tabular}{ccccccc}
\hline Caract/Esp. & $\begin{array}{c}\text { Bunodosoma } \\
\text { cangicum }\end{array}$ & $\begin{array}{c}\text { Anthopleura } \\
\text { cascaia }\end{array}$ & $\begin{array}{c}\text { Anthopleura } \\
\text { krebsi }\end{array}$ & $\begin{array}{c}\text { Anthopleura } \\
\text { sp. }\end{array}$ & Anemonia sp. & $\begin{array}{c}\text { Phyllactis } \\
\text { flosculifera }\end{array}$ \\
\hline $\begin{array}{c}\mathrm{N}^{\circ} \text { total de } \\
\text { tentáculos }\end{array}$ & 96 & 96 & 48 & 48 & $*$ & 48 \\
$\begin{array}{c}\text { Altura da } \\
\text { coluna }\end{array}$ & $\begin{array}{c}1,86 \pm 0,32 \\
(\mathrm{n}=7)\end{array}$ & $\begin{array}{c}0,32 \pm 0,13(\mathrm{n}) \\
=7)\end{array}$ & $\begin{array}{c}0,45 \pm 0,1 \\
(\mathrm{n}=4)\end{array}$ & $\begin{array}{c}0,36 \pm 0,14(\mathrm{n} \\
=3)\end{array}$ & $\begin{array}{c}0,21 \pm 0,05(\mathrm{n}= \\
6)\end{array}$ & $1,24 \pm 0,63(\mathrm{n}=4)$ \\
$\begin{array}{c}\text { Diâmetro } \\
\text { da coluna }\end{array}$ & $\begin{array}{c}2,6 \pm 1,2 \\
(\mathrm{n}=7)\end{array}$ & $\begin{array}{c}0,6 \pm 0,18 \\
(\mathrm{n}=7)\end{array}$ & $\begin{array}{c}0,8 \pm 0,2 \\
(\mathrm{n}=4)\end{array}$ & $\begin{array}{c}0,79 \pm 0,1 \\
(\mathrm{n}=3)\end{array}$ & $\begin{array}{c}0,53 \pm 0,17 \\
(\mathrm{n}=6)\end{array}$ & $\begin{array}{c}2,54 \pm 1,27 \\
(\mathrm{n}=4)\end{array}$ \\
$\begin{array}{c}\text { Diâmetro } \\
\text { do disco } \\
\text { oral }\end{array}$ & $\begin{array}{c}2,42 \pm 0,79 \\
(\mathrm{n}=7)\end{array}$ & $\begin{array}{c}0,41 \pm 0,16(\mathrm{n}) \\
=7)\end{array}$ & $\begin{array}{c}0,75 \pm 0,22(\mathrm{n}) \\
=4)\end{array}$ & $\begin{array}{c}0,84 \pm 0,17(\mathrm{n}) \\
=3)\end{array}$ & $\begin{array}{c}0,68 \pm 0,24 \\
(\mathrm{n}=7)\end{array}$ & $\begin{array}{c}2,91 \pm 1,04 \\
(\mathrm{n}=4)\end{array}$ \\
$\begin{array}{c}\text { Diâmetro } \\
\text { do disco } \\
\text { pedal }\end{array}$ & $\begin{array}{c}2,45 \pm 0,62 \\
(\mathrm{n}=7)\end{array}$ & $\begin{array}{c}0,61 \pm 0,27 \\
(\mathrm{n}=7)\end{array}$ & $\begin{array}{c}0,91 \pm 0,04(\mathrm{n}) \\
=4)\end{array}$ & $\begin{array}{c}0,85 \pm 0,2 \\
(\mathrm{n}=3)\end{array}$ & $\begin{array}{c}0,64 \pm 0,24 \\
(\mathrm{n}=8)\end{array}$ & $\begin{array}{c}2,5 \pm 0,68 \\
(\mathrm{n}=4)\end{array}$ \\
$\begin{array}{c}\text { N. exemp. } \\
\text { ex }\end{array}$ & 8 & 9 & 8 & 11 & 8 & 4 \\
\hline
\end{tabular}

*Não foi possível a contagem, pois os exemplares estudados estavam com os tentáculos contraídos.

Anatomia interna:

Anêmona com três ciclos de mesentérios: 12, 12, 24; com um total de 48 pares de mesentérios, dispostos regularmente, sendo dois pares de diretivos com músculos retratores bem visíveis e duas sifonoglifes. Presença de gônadas, porém pouco desenvolvidas nos exemplares estudados, presença de muitos filamentos mesentéricos.

Cnidoma:

No presente estudo foram observados e identificados os seguintes cnidocistos: espirocistos, holótrico, b-rabdóide e p-rabdóide A.

Tropical Oceanography, Recife, v. 33, n. 1, p. 19-31, 2005. 
Considerações sobre o habitat e a espécie:

As anêmonas desta espécie geralmente se encontram no mediolitoral entre fendas e frestas, em áreas parcialmente cobertas por sedimento, fixas em substrato rochoso, sujeita a correntes durante a mudança de marés. Como são muito aderentes e resistentes, torna-se difícil sua coleta. Quando expostas, permanecem completamente fechadas evitando a dessecação. As vesículas encontradas em sua coluna e o muco secretado por estas evitam a perda de água. No aquário do LAR/UFRPE, foi registrada a percepção do alimento pelos exemplares de Bunodosoma cangicum após alguns minutos da oferta de camarões, bem como a retração dos tentáculos da referida espécie na ausência da luz.

Anthopleura cascaia Corrêa, 1964 in Dube, 1981 (Figura 1B).

Distribuição geográfica:

Brasil - SP: Corrêa (1964); ES: Rosso (1984) apud Gomes (2002); BA: Dube (1974) e PE.

Pernambuco - Boa Viagem, Carneiros e Forno da Cal: Gomes (1996), Gomes \& Mayal (1997) e o presente estudo.

Morfologia externa:

Anêmona bem pequena. Disco pedal: circular, maior do que a coluna (diâmetro de 0,61 \pm 0,27 ) e bastante aderente, apresentando uma coloração bem clara, sendo parda ou esbranquiçada. Coluna: curta, achatada e lisa do meio da coluna para baixo (altura de $0,32 \pm 0,13$ ), na sua porção inferior bege, tornando-se marrom à medida que se aproxima do disco oral; próximas à margem, filas de verrugas que terminam em 48 projeções conforme também observado por Dube (1974) e que se apresentaram nas cores claras, esbranquiçadas e que aderem a pequenos cascalhos. Tentáculos: número total de 96, longos, finos, numerosos, esbranquiçados ou pardos e com manchas opacas e reflexos azulados. Disco oral: (diâmetro de 0,41 $\pm 0,16$ ), encontram-se esférulas marginais de coloração rosa clara que alternam com os tentáculos; a cor do disco oral varia de vermelha a marrom bastante escura; a boca é bem pequena e destacada na cor vermelha (Tabela 2).

Anatomia interna:

Anêmona com 10 pares de mesentérios ordinários perfeitos, 12 pares imperfeitos e 24 imperfeitos mais próximos ou estreitos, com muitos filamentos mesentéricos e gônadas não visíveis (exemplares muito pequenos), dois pares de diretivos com músculos retratores e com duas sifonoglifes visíveis à actinofaringe.

Cnidoma:

No presente estudo, foram observados e identificados: holótrico, b-rabdóide e p-rabdóide A. Considerações sobre o habitat e a espécie:

Encontrada no mediolitoral e fixa em substrato rochoso, em superfícies verticais, nas frestas entre algas. Durante a maré baixa, esta espécie se contrai e aderidos às suas verrugas estão numerosos cascalhos, garantindo proteção.

Anthopleura krebsi Duch. \& Mich., 1860 (Figura 1C).

Distribuição geográfica:

Mundo - Segundo Gomes (2002): EUA (Flórida e Texas), Caribe (Antilhas, Jamaica e Ilhas Virgens) e Brasil.

Brasil - ES: Gomes (1996) e PE.

Pernambuco - Fernando de Noronha: Belém \& Pinto (1990) e Pires et al. (1992), Boa Viagem, Piedade, Enseada dos Corais, Serrambi e Carneiros: Gomes (1996), Gomes \& Mayal (1997) e o presente estudo.

Morfologia externa:

Em alguns exemplares analisados, esta espécie se apresentou tanto com tamanho grande quanto pequeno. Disco pedal: muito aderente, arredondado e bem expansível, sendo mais largo que

Tropical Oceanography, Recife, v. 33, n. 1, p. 19-31, 2005. 
a coluna (diâmetro de 0,91 $\pm 0,04$ ), apresentando coloração creme clara, amarronzada ou parda. Coluna: (diâmetro de 0,8 $\pm 0,2$ ), cilíndrica, com coloração alaranjada ou esverdeada clara se aproximando do disco oral que é mais escurecido. Ao longo da coluna, encontram-se verrugas bem vermelhas ou marrons, às mesmas estão aderidos cascalhos, tornando a coluna muitas vezes curta ao se contrair, e na borda, esférulas bem verdes ou rosas que alternam com os tentáculos. Tentáculos: número total de 48, muitos, longos, finos e com manchas opacas, listradas ou não, com coloração clara esbranquiçada, parda ou opaca. Disco oral: (diâmetro de $0,75 \pm 0,22$ ) em algumas anêmonas estão bem distendidos na cor marrom ou verde escura e com uma boca central bem visível de coloração marrom (Tabela 2).

Anatomia interna:

Anêmona com três ciclos de mesentérios: 6, 6, 12, com um total de 24 pares de mesentérios dispostos geralmente de forma irregular. Dois pares de diretivos com músculos retratores e duas sifonoglifes. Presença de gônadas pouco desenvolvidas, mas não visíveis em todos os exemplares analisados e poucos filamentos mesentéricos.

Cnidoma:

No presente estudo, foram observados e identificados: espirocistos, holótricos, b-rabdóide e p-rabdóide A.

Considerações sobre o habitat e a espécie:

Esta espécie geralmente é encontrada reunida numa pequena agregação e vive entre frestas e pedras, evitando a exposição ao sol. Os fragmentos nesta aderidos servem de proteção contra a dessecação. A espécie A. krebsi, em condições desfavoráveis, tende a ficar estressada e se reproduz assexuadamente por fissão longitudinal (GOMES et al., 1998).

\section{Anthopleura sp.}

Distribuição geográfica:

Morfologia externa:

Brasil - Pernambuco (Boa Viagem).

Anêmona bem pequena. Disco pedal: muito aderente, de diâmetro um pouco maior que a coluna $(0,85 \pm 0,2 \mathrm{~cm})$, circular, com uma coloração vinho, porém mais clara que a coluna e às vezes com reflexos amarronzados. Coluna: (altura: 0,36 $\pm 0,14 \mathrm{~cm}$ ) em alguns exemplares curta, em outros um pouco mais comprida, de cor vinho com inúmeras verrugas que variam da cor vermelha a vinho. Tentáculos: número total de 48, muito finos, em alguns exemplares alongados, em outros curtos e com uma cor bem acentuada, vinho bem escura. Esférulas marginais na cor rosa ou esbranquiçadas, alternando com o ciclo de tentáculos. Disco oral: bem distendido, largo (0,84 $\pm 0,17$ $\mathrm{cm}$ ), na cor marrom ou vinho, com boca central na cor marrom bem clara (Tabela 2).

Anatomia interna:

Anêmona anatomicamente muito irregular, sem organização definida, com 20 pares de mesentérios imperfeitos e sete pares perfeitos, sendo três pares de diretivos e três sifonoglifes, com muitos filamentos e gônadas muito desenvolvidas em fileira e unidas aos filamentos mesentéricos e de coloração amarelada.

Cnidoma:

Foi identificado apenas o nematocisto b-rabdóide, os demais cnidocistos relatados para o gênero não foram observados, devido a difícil visualização.

Considerações sobre o habitat:

Encontrada em uma pequena população entre as locas e frestas nos bancos de arenito do mediolitoral da Praia de Boa Viagem e todos os espécimes de tamanho pequeno, provavelmente este é o tamanho máximo que esta espécie pode crescer.

Tropical Oceanography, Recife, v. 33, n. 1, p. 19-31, 2005. 
Anemonia sp.

Distribuição geográfica:

Brasil - Pernambuco (Forno da Cal, Itamaracá).

Morfologia externa:

Anêmona de tamanho pequeno a médio, com coloração variando de acinzentada à esverdeada. Disco pedal: nada aderente, com maior diâmetro do que a coluna $(0,64 \pm 0,24 \mathrm{~cm})$, com coloração um pouco avermelhada. Coluna: curta $(0,21 \pm 0,05 \mathrm{~cm})$, estreita $(0,53 \pm 0,17 \mathrm{~cm})$, lisa, avermelhada e/ou alaranjada. Tentáculos: com coloração esverdeada, finos, muitos, alguns maiores (os mais internos) e outros menores (os mais externos) de tamanho não uniforme e que não se retraem. Disco oral: bem distendido $(0,68 \pm 0,24 \mathrm{~cm})$, sempre bem aberto, com uma boca central e com reflexos esverdeados e acinzentados (Tabela 2).

Anatomia interna:

Não foi possível realizar o estudo quanto aos caracteres anatômicos, pois os exemplares não responderam adequadamente ao anestésico e, portanto, estavam muito contraídos.

Cnidoma:

No presente estudo, foram observados e identificados em Anemonia sp.: espirocisto, holótricos, b-rabdóide e p-rabdóide A.

Considerações sobre o habitat e a espécie:

As anêmonas desta espécie se encontravam no mediolitoral, bem soltas e de fácil coleta, na Praia de Forno da Cal, em Itamaracá (litoral Norte de Pernambuco). Estas se encontravam geralmente aderidas a esponjas ou ascídias. A sua musculatura é fraca, havendo pouca reação dos tentáculos a qualquer estímulo. Estas anêmonas passam a maior parte do tempo completamente distendidas, ficando susceptíveis à dessecação, porém esta espécie parece ser bastante resistente, sendo abundante em áreas poluídas, como as observadas em áreas de derrame de esgoto, na Pedra do Anel e do Jacaré, em Forno da Cal.

Phyllactis flosculifera (Lesueur, 1817) (Figura 1D).

Distribuição geográfica:

Mundo - Segundo Gomes (2002): Bahamas, Bermudas, Jamaica, Cuba, Haiti, Porto Rico, Saint Thomas, Barbados, Curaçao, Venezuela, Guadaloupe.

Brasil - BA: Dube (1974); ES: Belém \& Preslercravo (1973); RJ e SP: Corrêa (1964); SC: Schlenz \& Belém (1992) e PE.

Pernambuco - Boa Viagem, Enseada dos Corais, Pontal de Maracaípe, Carneiros e Muro Alto: Gomes (1996), Gomes \& Mayal (1997) e o presente estudo.

Morfologia externa:

Anêmona grande. Disco pedal: bastante expandido (diâmetro de $2,5 \pm 0,68$ ), pouco aderente e bem circular, com coloração alaranjada a avermelhada e com estrias avermelhadas que são as inserções mesenteriais. Coluna: com forma de cilindro e comprida; com coloração alaranjada, creme ou rosada e com estrias avermelhadas que são as inserções que dão continuidade desde o disco pedal até ao longo da coluna. Na margem, há uma série de 12 verrugas e 48 pseudoesférulas, conforme observado também por Schlenz \& Belém (1992). O parapeito ou colarinho é composto de pequenas vesículas verdes, fazendo com que se pareça com um tapete. Próximo da região do colarinho ocorre uma coloração azul a lilás. Tentáculos: número total de 48, longos, finos e pontiagudos de cor bege clara ou parda e com manchas brancas, estes tentáculos se localizam entre o parapeito e o centro do disco oral. Disco oral: bem estreito com uma boca no centro, com uma cor esverdeada escura (Tabela 2).

Anatomia interna:

Anêmona com 10 pares de mesentérios ordinários perfeitos, 12 de mesentérios imperfeitos e dois pares de diretivos seguidos de duas sifonoglifes, complementando ao todo 24 pares de 
mesentérios, músculos retratores, poucos filamentos mesentéricos e gônadas com um pequeno desenvolvimento.

Cnidoma:

No presente estudo, foram observados e identificados: espirocistos, b-rabdóide e prabdóide A.

Considerações sobre o habitat e espécie:

Essa anêmona geralmente vive enterrada em substrato arenoso ou em locas, apenas com o disco oral expandido. A cor esverdeada encontrada no colarinho se deve à abundância de zooxantelas. Essa cor do colarinho serve muitas vezes de camuflagem para outros animais (GOMES, 1996).

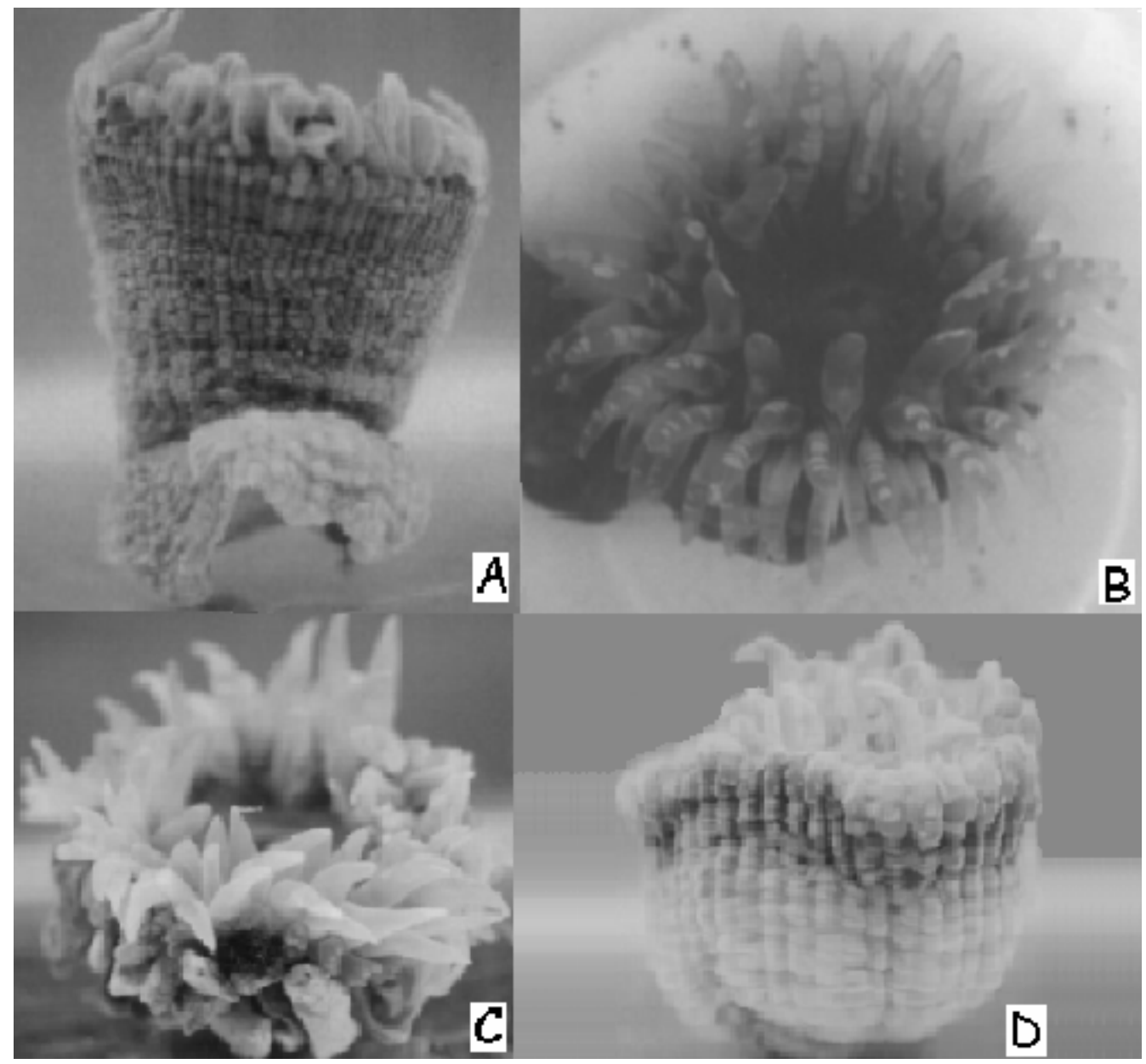

Figura 1 - Anêmonas identificadas em algumas praias do litoral pernambucano. A) Bunodosoma cangicum Corrêa, 1964 in Belém \& Preslercravo, 1973. Foto: Fernando Azevedo; B) Anthopleura cascaia Corrêa, 1964 in Dube, 1981. Foto retirada de Gomes (1996); C) Anthopleura krebsi Duch. \& Mich., 1860. Foto: Fernando Azevedo; D) Phyllactis flosculifera (Lesueur, 1817). Foto: Fernando Azevedo. Escalas: 2:1cm.

Tropical Oceanography, Recife, v. 33, n. 1, p. 19-31, 2005. 


\section{DISCUSSÃO}

A contribuição do presente estudo reside, principalmente, nos dados inéditos do levantamento da anemonofauna de Muro Alto e Forno da Cal, além da morfometria dos caracteres externos estudados preliminarmente nas seis espécies de anêmonas identificadas.

Em relação à comparação com outros estudos recentes da anemonofauna do estado de Pernambuco, Gomes (2002) relatou 17 espécies de actiniarias, porém foram estudadas 10 localidades pela referida autora. Considerando ainda, dentro do nosso Estado o Arquipélago de Fernando de Noronha, onde foram registradas sete espécies por Pires et al. (1992), sendo que apenas uma, a Anthopleura krebsi foi coincidente com o presente estudo, devido provavelmente ao isolamento geográfico e as diferentes condições ecológicas do referido Arquipélago.

A anemonofauna que apresentou maior riqueza de espécies foi a da Praia dos Carneiros, vindo em seguida a da Praia de Boa Viagem, com quatro espécies ocorrentes; Muro Alto e Forno da Cal que se apresentaram cada uma com três das espécies identificadas.

A maior riqueza de espécies de actiniarias da Praia dos Carneiros foi também relatada por Gomes (1996) que registrou além das espécies inventariadas para o presente estudo, as actiniarias: Psamanthus caraguaensis Rosso, 1984, Anemonia sargassensis Hargitt, 1908, Actinia bermudensis (Mc Murrich, 1889) e Stichodactyla duerdeni Carlgren, 1900, antes conhecida como Homostichanthus duerdeni. Esta praia apresentou maior número de indivíduos em relação às demais, possivelmente, devido à distância que se encontra dos grandes centros urbanos e a dificuldade do acesso que as pessoas encontram. Além das espécies identificadas no presente trabalho, foram ainda observadas anêmonas do gênero Anthopleura e aconciarias, do gênero Bellactis, o que concorda com Pinto (2002) que coletou vários exemplares de Bellactis ilkalyseae Dube, 1983 na praia acima citada.

Em Boa Viagem, a distribuição pode estar associada a fatores ambientais, como poluição pelas descargas de esgotos dos prédios e das casas próximos ao mar, neste local as espécies ocorrentes dos gêneros Anthopleura e Bunodosoma apresentaram, aparentemente, uma grande resistência a estes tipos de ambientes.

Em Muro Alto, as espécies Bunodosoma cangicum, Phyllactis flosculifera e Anthopleura krebsi se distribuíam nas superfícies dos bancos de arenito, próximos a vários hotéis, podendo este fator interferir na distribuição dessas espécies.

A espécie Anemonia sp. ocorreu apenas em Forno da Cal, local bastante poluído pela ação do homem, através de derrames de lixos e de esgotos de casas de veraneio próximos ao mar, contudo, esta espécie juntamente com as espécies Bunodosoma cangicum e Anthopleura cascaia apresentaram grande resistência a estes fatores poluentes, como anteriormente citado em Muro Alto. Além disso, a espécie Anemonia sp. foi observada crescendo em cima de ascídias e esponjas, fator este que pode estar relacionado a algum tipo de associação, em que a anêmona utiliza a esponja ou a ascídia como um substrato, podendo assim ser considerada epizóica.

Essa variabilidade na anemonofauna pode estar relacionada aos parâmetros físico-químicos dependendo do local, tais como: temperatura, salinidade, mudança de marés, $\mathrm{pH}$, profundidade, tipo de substrato.

Quanto aos caracteres morfológicos estudados nas espécies inventariadas, os resultados obtidos foram semelhantes quando comparados às demais literaturas, no entanto ocorreu alguma variação no que diz respeito à coloração e ao tamanho das estruturas das espécies identificadas. Estas diferenças ocorreram, possivelmente, devido a fatores físicos do ambiente, o que já foi mencionado anteriormente, pelo tempo prolongado dos exemplares no aquário do LAR/UFRPE, pela presença de zooxantelas ou ainda por uma fixação não perfeita do animal. A maioria dos resultados quanto à anatomia e à identificação do cnidoma corroboraram com vários autores.

Tropical Oceanography, Recife, v. 33, n. 1, p. 19-31, 2005. 
A espécie Anthopleura sp. assemelhou-se bastante quanto à disposição das estruturas e coloração quando comparadas as literaturas de Corrêa (1964) e Dube (1974) onde estas autoras descreveram Anthopleura cascaia com uma coloração vermelha, distribuída na coluna e nos tentáculos. Quanto aos caracteres anatômicos, por outro lado, esta espécie acima referida aproximou-se à espécie Anthopleura varioarmata Watzl, 1922, descrita no trabalho de Belém \& Monteiro (1984), referente à irregularidade de conter no mínimo três sifonoglifes e três pares de diretivos, reproduzir-se em aquário facilmente e apresentar gônadas visíveis, contudo são necessários estudos mais aprofundados dos cnidocistos para se ter a confirmação precisa. Essa variabilidade na anatomia pode estar associada à reprodução por fissão longitudinal, segundo Belém \& Pinto, 1990.

Com relação a Anemonia sp., os exemplares estudados quanto à morfologia assemelham-se a A. sargassensis Hargitt, 1908, com relação às estruturas e à coloração da coluna vermelha, citadas por Corrêa (1964), Dube (1974) e Gomes (1996). Por outro lado, a ausência de pregas na coluna quando contraídas citadas por Corrêa (1964) e Dube (1974), diferencia morfologicamente a Anemonia sp. de Anemonia sargassensis. O estudo mais detalhado da anatomia e dos cnidocistos não foi possível, impossibilitando a identificação até o nível específico desta espécie.

\section{CONCLUSÃO}

- Foram inventariadas e coletadas seis espécies, para as Praias de Boa Viagem, Muro Alto, Carneiros e Forno da Cal, porém o levantamento da biodiversidade das anêmonas de Pernambuco ainda se encontra em um estágio inicial (especialmente em relação ao gênero Anthopleura), torna-se necessário o levantamento das espécies de actiniarias nos recifes submersos e no mediolitoral de outras praias do litoral Sul e ainda nas praias não estudadas, como as do Setor Norte, entre estas: Ponta de Pedras, Carne de Vaca e Catuama.

- Em relação à morfologia dos caracteres estudados, estes corroboraram com os dados da literatura, com exceção da coloração e do tamanho dos exemplares estudados, pois estes apresentaram uma grande variabilidade. Para o cnidoma das anêmonas pesquisadas, encontrouse um padrão semelhante aos já registrados anteriormente por outros estudiosos.

- Estudos posteriores podem ser planejados, como a caracterização completa da histologia e do cnidoma, além da utilização de técnicas para a extração de DNA para a identificação de Anemonia sp. e Anthopleura sp. até o nível específico, o que provavelmente resultará em duas espécies novas para a ciência. Com base nos resultados presentes e da bibliografia já existente, deve-se pensar agora em uma revisão dos gêneros supracitados, além do estudo de substâncias bioativas e respostas a variações abióticas (mais especificamente poluição) das espécies de taxonomia já conhecida, como B. cangicum.

\section{AGRADECIMENTOS}

Ao PIBIC/ CNPq/UFRPE por ter auxiliado no desenvolvimento deste trabalho através da concessão de bolsa de Iniciação Científica para a primeira autora. Às Doutoras Suzana Pinto da Universidade de São Paulo e Paula Braga Gomes, pesquisadora da Universidade Federal de Pernambuco, por terem auxiliado nos estudos das anêmonas; ao fotógrafo Fernando Azevedo da Universidade Federal Rural de Pernambuco e aos demais pesquisadores que de forma direta ou indireta contribuíram para a execução deste trabalho.

Tropical Oceanography, Recife, v. 33, n. 1, p. 19-31, 2005. 


\section{REFERÊNCIAS BIBLIOGRÁFICAS}

ALVES, M. S. Estrutura da macroepifauna vágil no ecossistema Halodule wrightii da Ilha de Itamaracá, Nordeste do Brasil. São Paulo, 2000. 112p. Tese de Doutorado. Instituto Oceanográfico. Universidade de São Paulo.

AMARAL, F.D. Agaricia agaricites (Linné) humilis, Verrill, 1901, Favia gravida (Verrill, 1868) e Montastrea cavernosa (Linné, 1766) do litoral Sul de Pernambuco (Região de Tamandaré). Recife. 1986. 66p. Monografia de Bacharelado em Biologia da UFPE. Universidade Federal de Pernambuco.

AMARAL, F. D.; HUDSON, M. M.; SILVEIRA, F. L.; MIGOTTO, A.; PINTO, S. M.; LONGO, L. Cnidarians of Saint Peter and St. Paul Archipelago, Northeast Brazil. In: INTERNATIONAL CORAL REEF SYMPOSIUM. 9, 2000, Bali, Proccedings... Bali: 2000. p. 567-572.

AMARAL, F. D.; SILVEIRA, S. R. M.; STEINER, A. Q.; SANTOS, M. G.; COSTA, C. F.; MELO, K. V.; BARRADAS, J. I.; LEMOS, S.; HUDSON, M.; ESTEVES, E.; LEAL, F.; MARQUES, L.; VASCONCELOS, S.; RAMOS, M.; XAVIER, M.; NEVES, R.; OLIVEIRA, J.; SOUZA, C. A.; SENA, F.; MACEDO, A.; BARROS, T.; ROCHA, A. Atividades de extensão do Laboratório de Ambientes Recifais (LAR/UFRPE). In: CONGRESSO BRASILEIRO DE EXTENSÃo UNIVERSITÁRIA, 1, 2003, João Pessoa, Anais... João Pessoa: 2003, CD-ROM. P. 18.

AMARAL F. D.; STEINER, A. Laboratório de Ambientes Recifais. Net, Recife, 2002. Disponível em <http://www.ufrpe.br/lar/index.htmlT>. Acesso em 20 outubro de 2002.

BELÉM, M. J. C.; MONTEIRO, D. C. Fauna de cnidários do Rio de Janeiro. III. Anthopleura varioarmata Waltz, 1922 (Actiniaria, Endomyaria), uma nova ocorrência de Actiniidae. In:

Seminário de Biologia Marinha, Academia Brasileira de Ciências. Rio de Janeiro. p. 193-203. 1984.

BELÉM, M. J. C.; PRESLERCRAVO, J. C. Contribuições ao conhecimento da fauna de cnidários do Espírito Santo, Brasil I - Considerações sobre Actiniaria do Município de Aracruz, E. S. Série Zoologia, Espírito Santo. v. 1, n. 80, p. 2-17. 1973.

BELÉM, M. J. C.; PINTO, S. M. Morphological and microanatomical study of Anthopleura krebsi Duchassaing \& Michelotti, 1860 (Cnidaria, Anthozoa, Actiniidae). A new record in Brazil. Anais da Academia Brasileira de Ciência. Rio de Janeiro. v. 62, n. 2, p. 183-192. 1990

CORRÊA, D. D. Corallimorpharia e Actiniaria do Atlântico Oeste Tropical. São Paulo, 1964. 139p. Tese (Prof. Catedrático). Universidade de São Paulo.

DUBE, V. M. Anêmonas do Mar (Ordem Actiniaria) do estado da Bahia. Salvador, 1974. 81p. Dissertação de Mestrado. Universidade Federal da Bahia.

FAUTIN, D. G. E ALLEN, G. R. Field guide to anemone fishes and their host sea anemones. California Academy of Sciences, Western Australian Museum, 1992. 160p.

Tropical Oceanography, Recife, v. 33, n. 1, p. 19-31, 2005. 
GOMES, P. B. Anêmonas do Mar (Actiniaria, Anthozoa, Cnidaria) do litoral de Pernambuco. Recife. 1996. 52 p. Monografia de Bacharelado. Universidade Federal de Pernambuco.

GOMES, P. B. Anêmonas-do-Mar (Cnidaria, Actiniaria) de Pernambuco (Brasil). In: Tabarelli, M.; Silva, J. M. C. (eds.). Diagnóstico da Biodiversidade de Pernambuco. Recife: Editora Massangana. vol. 2. p. 357-363. 2002.

GOMES, P. B.; BELÉM, M. J.; SCHLENZ, E. Distribution, abundance and adaptations of three species of Actiniidae (Cnidaria, Actiniaria) on an intertidal beach rock in Carneiros beach, Pernambuco, Brazil. Miscellània Zoològica. v. 21, n. 2, p. 65-72. 1998

GOMES, P. B.; MAYAL, E. Histórico dos Estudos das Anêmonas do Mar (Cnidaria, Actiniaria) no Brasil. Trabalhos Oceanográficos da Universidade Federal de Pernambuco. Recife. v. 25, p. 111-119. 1997.

GOMES, P. B.; ZAMPONI, M. O. Registro de Phyllactis correae Schlenz \& Belém, 1988 (Actiniaria, Actiniidae) para o litoral de Pernambuco, Brasil. Biociências. Porto Alegre, v. 6, n. 2, p. 185-187. 1998.

MIGOTTO, A. E.; SILVEIRA, F. L.; SCHLENZ, E.; FREITAS, J. C. Filo Cnidaria. In: Migotto, A. E.; Tiago, C. G. (Eds.). Biodiversidade do Estado de São Paulo - Síntese do conhecimento ao final do século XX., São Sebastião: Centro de Biologia Marinha - USP, 1999. v. 3, p. 36-46.

PINTO, S. M. Anêmonas-do-mar com acôncios (Anthozoa: Actiniaria, Mesomyaria). Análise morfológica e molecular. São Paulo, 2002. 186p. Tese de Doutorado. Universidade de São Paulo.

PIRES, D. O.; CASTRO, C. B.; MIGOTTO, A. E.; MARQUES, A. C. Cnidários bentônicos de Fernando de Noronha, Brasil. Boletim do Museu Nacional do Rio de Janeiro. Rio de Janeiro. n. 354, p. 1-21. 1992.

SCHMIDT, H. On Evolution in the Anthozoa. In: INTERNATIONAL CORAL REEF SYMPOSIUM, 2, 1974, Brisbane, Proceedings... Brisbane: 1974. p. 533-560.

SCHLENZ, E. E BELÉM, M. J. C. Phyllactis correae. n. sp. (Cnidaria, Actiniidae) from Atol das Rocas, Brasil, with notes on Phyllactis flosculifera (Lesueur,1817). Boletim da Universidade de São Paulo. São Paulo. v. 12, p. 91-117. 1992.

STEINER, A. Q. Percepção ambiental, etnoecologia e educação ambiental: experiências acerca do tema “ambientes recifais”. Recife. 2003. 141p. Monografia de Bacharelado. Universidade Federal Rural de Pernambuco. 